\title{
Pediatric Asthma Caregiver's Quality of Life Questionnaire is a useful tool for monitoring asthma in children
}

\author{
Iwona Stelmach • Daniela Podlecka $\cdot$ Katarzyna Smejda • \\ Paweł Majak · Joanna Jerzyńska $\cdot$ Rafał Stelmach $\cdot$ \\ Anna Janas $\cdot$ Włodzimierz Stelmach
}

Accepted: 17 November 2011 / Published online: 4 December 2011

(C) The Author(s) 2011. This article is published with open access at Springerlink.com

\begin{abstract}
Purpose There is little agreement among researchers whether a caregiver's QOL can be used to detect changes in asthma severity in children. We assessed correlation between QOL in parents and QOL in children with asthma as well as clinical parameters of asthma. We determined whether changes in caregiver's QOL scores reflect changes in child's QOL and their asthma control.

Methods This was a 9-week period cohort study. One hundred and ten primary caregivers with 110 children were seen in the clinic at enrollment, at week \#1, \#5 and \#9. At each visit, the parents completed the Pediatric Asthma Caregiver's Quality of Life Questionnaire (PACQLQ).

Results One hundred and one children and 101 caregivers completed the study. We found a significant correlation between asthma diary score in children and QOL in parents (from $r=-0.46$ to $r=-0.53$ ). We also found significant positive correlation between PAQLQ and PACQLQ and significant association between changes in asthma control and PACQLQ score for both domains. We observed significant change in PACQLQ of caregivers whose children obtained asthma control.
\end{abstract}

I. Stelmach $(\bowtie) \cdot$ D. Podlecka $\cdot$ K. Smejda $\cdot$ P. Majak ·

J. Jerzyńska

Department of Pediatrics and Allergy, Medical University

of Lodz, N. Copernicus Hospital,

62 Pabianicka Str., 93-513 Lodz, Poland

e-mail: alergol@kopernik.lodz.pl

R. Stelmach · A. Janas

Institute of Dental Surgery, Faculty of Medicine and Dentistry,

Medical University of Lodz, Lodz, Poland

W. Stelmach

Department of Social and Preventive Medicine, Medical

University of Lodz, Lodz, Poland
Conclusions PACQLQ is a useful tool for monitoring asthma in children. The implementation of the PACQLQ would be helpful in involving parents in therapy of their children with asthma.

Keywords Quality of life · Parents · Children · Asthma

\section{Introduction}

Asthma can impair parents and primary caregivers of children with asthma normal daily activities. Pediatric Asthma Caregiver's Quality of Life Questionnaire (PACQLQ) by Juniper et al. [1] refers to these impairments. We have previously validated Pediatric Asthma Quality of Life Questionnaire (PAQLQ) among asthmatic children in Poland [2]. For the purpose of the present study, we assessed correlation between asthma quality of life in parents and asthma quality of life in children as well as clinical parameters of asthma in children. We determined whether changes in caregiver's QOL scores reflect changes in child's QOL and their asthma severity such as clinical asthma control and physical indicators of inflammation: FENO and $\triangle F E V 1$. We tried to answer what change in the caregiver's QOL score can be considered important.

\section{Materials and methods}

Subjects

We reanalyzed the clinical data of 110 children with atopic asthma from our PAQLQ validation study; the details of the study were previously described [2]. One hundred and ten primary caregivers (age 24-58 years; $80 \%$ female), 
usually a parent, who lived with the child at least $75 \%$ of the time, accompanied each child to every clinic visit and were able to communicate directly with the interviewer. They all signed an informed consent. The study was approved by the Ethics Committee of the Medical University of Lodz, Poland.

\section{Study design}

This was a 9-week period cohort study. The caregivers and children were seen in the clinic at enrollment (visit 1), at week \#1 (visit 2), \#5 (visit 3) and \#9 (visit 4), and they completed the PACQLQ [3]. Each child received an asthma diary and a portable peak flow meter for recording morning PEFR at home 1 week before each follow-up clinic visit. At each clinic visit, spirometry before and 20 min after bronchodilator administration and NO measurements were performed in all children.

\section{Pediatric Asthma Caregiver's Quality of Life}

Questionnaire

Quality of life of the parents/caregivers of the children was assessed using PACQLQ by Juniper et al. [1]. It is a selfadministered instrument, which includes 13 items (4 concern activity limitations and 9 concern emotional function). Responses to each item of the PACQLQ are given on a seven-point scale, ranging from 1 to 7 , with the higher scores indicating less impairment. The result was expressed as a mean score per item for each of the domains, as well as for the overall quality of life [1].

\section{Pediatric Asthma Quality of Life Questionnaire (PAQLQ)}

Health-related quality of life was assessed by the Polish version of PAQLQ by Juniper et al. [3].

Asthma control evaluation

A child was considered either under control or not under control in week \#1 and \#5 according to the guidelines [4]. We considered asthma to be inadequately controlled when one or more of the following were present: awoken at night by symptoms, persistent waking in the mornings with symptoms, limitation of normal daily activities and use of inhaled short-acting bronchodilator use more than two puffs a day. The patients whose asthma was not adequately controlled at week \#1 or \#5 were referred to their asthma doctor to increase their controller medications. We categorized children as having asthma control: (1) stable-if their asthma control did not change between visit 2 and 4 , (2) lost - if their asthma was not controlled at visit 4 and was controlled at visit 2, (3) obtained-if their asthma was controlled at visit 4 and was not controlled at visit 2 .

Child's asthma diary

The asthma diary card included daytime symptoms (completed at bedtime), incidents of nocturnal awakening (recorded in the morning upon awakening) and the number of puffs of as-needed $\beta 2$-agonist [2]. The minimum score for each day was 0 (no symptoms during the daytime, no symptoms at night and no use of $\beta 2$-agonists). The maximum score was 36 (severe symptoms during the day and night, and the usage of the $\beta 2$-agonists more frequent than 16 puffs most days).

Lung function measurements

Pulmonary function testing in each child was performed with a Master Screen unit (Erich Jaeger Gmbh-Hochberg, Germany). Flow-volume curves were performed according to American Thoracic Society standards [5].

Nitric oxide measurement

The NO measurements were performed according to ERS/ $\mathrm{ATS}^{1}$ with a chemiluminescence analyzer (model 280i nitric oxide analyzer; Sievers, Boulder, CO, USA) and defined in parts per billion.

Statistical analysis

Spearman's rank correlation coefficients were used to assess relations between PACQLQ score and clinical parameters and PAQLQ in children. To assess changes in PACQLQ score separately for caregivers whose children had stable or lost/obtained asthma control between visits, Wilcoxon single sample test was used (with 0 as a comparison value). All statistical calculations were performed using StatSoft Statistica for Windows, release 6.0 (StatSoft, Inc., Tulsa, USA). A $P$ value of $<0.05$ was considered statistically significant.

\section{Results}

One hundred and one children and 101 caregivers completed the study; 9 patients and 9 caregivers withdrawn the consent before week \#1.

\footnotetext{
${ }^{1}$ Recommendations for standardized procedures for the on-line and off-line measurement of exhaled lower respiratory nitric oxide and nasal nitric oxide in adults and children-1999. This official statement of the American Thoracic Society was adopted by the ATS Board of Directors, July 1999 (1999). American Journal of Respiratory and Critical Care Medicine, 160, 2104-2117.
} 
We found a significant statistical correlation between asthma diary score in children and asthma quality of life in parents (from $r=-0.46$ to $r=-0.53$ ) (Table 1). These findings were consistent in both domains: activities and emotional. We did not observe any significant correlation between other clinical parameters and asthma quality of life in parents. We found significant positive correlation between PAQLQ and PACQLQ in all domains (Table 1).

We also analyzed prospectively changes in PACQLQ in relation to all clinical parameters as well as PAQLQ (Table 2). We found negative correlation between changes in PACQLQ and asthma diary in children; improvement in caregivers quality of life correlates with better asthma control in children. Changes in PACQLQ correlated positively with changes in PAQLQ.

Table 1 Correlation between PACQLQ score and clinical parameters and PAQLQ in children at visit $2-4$

\begin{tabular}{|c|c|c|c|}
\hline & \multicolumn{3}{|c|}{ Asthma quality of life score in parent } \\
\hline & Activities & Emotions & Total \\
\hline \multicolumn{4}{|l|}{ Visit 2} \\
\hline Asthma diary & $-0.45^{* *}$ & $-0.49 * *$ & $-0.49 * *$ \\
\hline FeNO & 0.57 & 0.60 & 0.62 \\
\hline 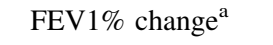 & 0.03 & -0.01 & -0.01 \\
\hline FEV1 & -0.01 & 0.02 & 0.03 \\
\hline PEF & 0.15 & 0.01 & 0.06 \\
\hline PAQLQ symptoms & $0.57 * *$ & $0.60 * *$ & $0.62 * *$ \\
\hline PAQLQ activities & $0.48 * *$ & $0.50 * *$ & $0.51 * *$ \\
\hline PAQLQ emotions & $0.44 * *$ & $0.40 * *$ & $0.43 * *$ \\
\hline \multicolumn{4}{|l|}{ Visit 3} \\
\hline Asthma diary & $-0.45 * *$ & $-0.45 * *$ & $-0.46 * *$ \\
\hline FeNO & 0.52 & 0.51 & 0.52 \\
\hline 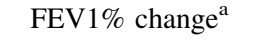 & -0.02 & -0.01 & -0.02 \\
\hline FEV1 & -0.02 & 0.06 & 0.04 \\
\hline PEF & 0.19 & 0.08 & 0.11 \\
\hline PAQLQ symptoms & $0.52 * *$ & $0.51 * *$ & $0.52 * *$ \\
\hline PAQLQ activities & $0.46 * *$ & $0.48 * *$ & $0.49 * *$ \\
\hline PAQLQ emotions & $0.46 * *$ & $0.45^{* *}$ & $0.47 * *$ \\
\hline \multicolumn{4}{|l|}{ Visit 4} \\
\hline Asthma diary & $-0.49 * *$ & $-0.53 * *$ & $-0.53 * *$ \\
\hline FeNO & 0.56 & 0.59 & 0.59 \\
\hline 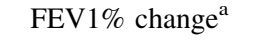 & 0.02 & -0.01 & -0.01 \\
\hline FEV1 & -0.04 & -0.08 & -0.06 \\
\hline PEF & 0.15 & 0.14 & 0.14 \\
\hline PAQLQ symptoms & $0.56 * *$ & $0.59 * *$ & $0.59 * *$ \\
\hline PAQLQ activities & $0.40 * *$ & $0.45 * *$ & $0.45^{* *}$ \\
\hline PAQLQ emotions & $0.46 * *$ & $0.50 * *$ & $0.50 * *$ \\
\hline
\end{tabular}

Data are presented as correlation coefficients (Spearman rank)

$* P<0.05 ; * * P<0.01$

a $\%$ change from baseline in bronchial reversibility test
Table 2 Correlation between changes in PACQLQ and changes in clinical parameters and PAQLQ in children

\begin{tabular}{lccc}
\hline Change between visit 2 and 4 in: & $\begin{array}{l}\text { Asthma quality of life score in } \\
\text { parent (change between visit } 2 \\
\text { and 4) }\end{array}$ \\
\cline { 2 - 4 } & Activities & Emotions & Total \\
\hline Asthma diary & $-0.34^{* *}$ & $-0.32^{* *}$ & $-0.34^{* *}$ \\
FeNO & 0.37 & 0.36 & 0.38 \\
FEV1\% change & -0.08 & -0.13 & -0.15 \\
FEV1 & -0.03 & -0.03 & -0.02 \\
PEF & 0.28 & 0.13 & 0.18 \\
PAQLQ symptoms & $0.37^{* *}$ & $0.36^{* *}$ & $0.38^{* *}$ \\
PAQLQ activities & $0.24^{*}$ & $0.41^{* *}$ & $0.39^{* *}$ \\
PAQLQ emotions & $0.23^{*}$ & $0.29^{* *}$ & $0.27^{* *}$ \\
\hline
\end{tabular}

Data are presented as correlation coefficients (Spearman rank)

$* P<0.05 ; * * P<0.01$

a $\%$ change from baseline in bronchial reversibility test

Table 3 Changes in PACQLQ in comparison with the changes in the level of asthma control

Change between visit 2 and Change in the level of asthma control 4 in: between 2 and 4 visit

\begin{tabular}{lll} 
& $\begin{array}{l}\text { Lost asthma } \\
\text { control }\end{array}$ & $\begin{array}{l}\text { Obtained asthma } \\
\text { control }\end{array}$ \\
\hline PACQLQ ${ }^{\text {a }}$ activities & $0(-3$ to 1$)$ & $2(0 \text { to } 4.5)^{* *}$ \\
PACQLQ emotions & $0(-7$ to 12$)$ & $6(0.5 \text { to } 11)^{* *}$ \\
Total PACQLQ score & $1(-10$ to 14$)$ & $8(0.5 \text { to } 16)^{* *}$ \\
\hline$* P<0.05 ; * * P<0.01$ & & \\
a Asthma quality of life score in parent &
\end{tabular}

We analyzed changes in PACQLQ score in relation to asthma control in children. We also analyzed changes in PACQLQ score separately for caregivers whose children had stable or lost/obtained asthma control between visits. We observed significant change in PACQLQ of caregivers whose children obtained asthma control, but there was no significant change when children lost asthma control (Table 3).

\section{Discussion}

The results of this study suggest that the PACQLQ performs well in clinical practice. The questionnaire detects differences in quality of life in caregivers with different degrees of quality of life and asthma symptoms in children. We found significant positive correlation between PAQLQ and PACQLQ in all domains. What is interesting is the correlation between changes in asthma symptoms in children is greater with PACQLQ than with PAQLQ 
( $r=-0.34$ vs. $r=-0.22$ ), which suggest that measuring quality of life in parents could be a useful tool in monitoring asthma in children [2]. What is more important is measuring quality of life in caregivers, which may reflect current asthma control in children. The important finding of our study is that PACQLQ reflects better obtained asthma control in children than lost control. It seems obvious that more impaired quality of life in parents as a result of their child's asthma affects all aspects of family life and increase a total cost of asthma management.

It is interesting that our study showed a significant statistical correlation between asthma diary score in children and asthma quality of life in parents. However, Merikallio et al. [6] demonstrated in their study that a short-time QOL can be obtained from children, but for longitudinal observation parents' information seems to be more adequate. In opposite to the study of Farnik et al. [7], we found significant positive correlation between PAQLQ and PACQLQ in all domains. We did not observe any significant correlation between clinical parameters in children and asthma quality of life in parents, which stay in accordance with previous studies [7]. We interpret these results as clinical parameters such as FEV1 and FeNO being weak indicators of disease severity especially in short-time observation of mild asthma. However, other studies have shown the correlation between children's lung function and parental QOL questionnaire [7]. The most impaired parent domain-emotions significantly correlated with FEV1\%FVC. Even if the correlations between clinical parameters and PACQLQ are weak or none, it should not determine a limitation of this questionnaire in the management of child's asthma.

This study also showed that the parent's assessment of change in quality of life was more closely related to asthma diary scores in children than child's assessment. Although this is not a diagnostic scale, it should be assessed as complement to traditional clinical evaluation.

We found significant association between changes in asthma control and PACQLQ score for both domains. We observed significant change in PACQLQ of caregivers whose children obtained asthma control, but there was no significant change when children lost asthma control. In contrary to our results, Farnik et al. [7] showed that uncontrolled asthma is associated with a reduced HRQOL in pediatric caregivers.

The limitation of the study is related to lack of other aspects of parent's characteristics, for example, parental education or financial situation. However, in the study of Farnik et al. [8], occupational domain in QOL in Child's Chronic Disease Questionnaire of parents did not show any significant differences of stable and unstable children with chronic disease.

The implementation of the PACQLQ was successful in our study and would be helpful in involving parents in therapy of their children with asthma.

Acknowledgments This study was funded by grant 502-12-760 and 503-2056-1 from the Medical University of Lodz, Poland.

Conflict of interest Authors declare no conflict of interest.

Open Access This article is distributed under the terms of the Creative Commons Attribution Noncommercial License which permits any noncommercial use, distribution, and reproduction in any medium, provided the original author(s) and source are credited.

\section{References}

1. Juniper, E. F., Guyatt, G. H., Ferrie, D. H., Ferrie, P. J., Griffith, L. E., \& Townsed, M. (1996). Measuring quality of life in the parents of children with asthma. Quality of Life Research, 5, 27-34.

2. Stelmach, I., Podlecka, D., Majak, P., Jerzyńska, J., Stelmach, R., \& Janas, A., et al. (accepted for publication). Validity of the pediatric asthma quality of life questionnaire in polish children. Pediatric Allergy and Immunology.

3. Juniper, E. F., Guyatt, G. H., Ferrie, D. H., Ferrie, P. J., Griffith, L. E., \& Townsed, M. (1996). Measuring quality of life in children with asthma. Quality of Life Research, 5, 35-46.

4. Joint Task Force on Practice Parameters, American Academy of Allergy, Asthma and Immunology and Joint Council of Allergy, \& Asthma and Immunology. (2005). Attaining optimal asthma control: a practice parameter. The Journal of Allergy and Clinical Immunology, 116(5), S3-S11.

5. Miller, M. R., Hankinson, J., Brusasco, V., Burgos, F., Casaburi, R., Coates, A., et al. (2005). Standardisation of spirometry. European Respiratory Journal, 26, 319-338.

6. Merikallio, V. J., Mustalahti, K., Remes, S. T., Valovirta, E. J., \& Kalia, M. (2005). Comparison of quality of life between asthmatic and healthy school children. Pediatric Allergy \& Immunology, 16, 332-340.

7. Farnik, M., Pierzchała, W., Brożek, G., Zejda, J., \& Skrzypek, M. (2010). Quality of life protocol in the early asthma diagnosis in children. Pediatric Pulmonology, 45, 1095-1102.

8. Farnik, M., Brożek, G., Pierzchała, W., Zejda, J., Skrzypek, M., \& Walczak, Ł. (2010). Developement, evaluation and validation of a new instrument for measurement quality of life in the parents of children with chronic disease. Health and Quality of Life Outcomes, 8, 151. 